\title{
Syntheses of diazadithiacrown ethers containing two 8- hydroxyquinoline side arms
}

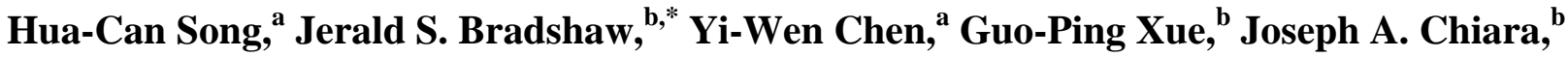 \\ Krzysztof E. Krakowiak, ${ }^{b}$ Paul B. Savage, ${ }^{b}$ Zun-Le Xue, ${ }^{a}$ and Reed. M. Izatt ${ }^{b}$ \\ Department of Chemistry, Zhongshan University, Guangzhou 510275 P. R. China ${ }^{b}$ Department of \\ Chemistry \& Biochemistry, Brigham Young University, Provo, UT 84602, USA \\ E-mail: Jerald_bradshaw@byu.edu
}
This paper is dedicated to Professor Miha Tišler to mark his $75^{\text {th }}$ birthday and to acknowledge his dedication to synthetic heterocyclic chemistry
(received 09 Mar 01; accepted 31 Jul 01; published on the web 08 Aug 01)

\begin{abstract}
Ten new diazadithiacrown ethers containing two 8-hydroxyquinoline (HQ) sidearms attached through the HQ 7-positions and four new diazadithiacrown ethers containing two HQ sidearms attached through the HQ 2-positions have been prepared. Some of these new ligands also contain a hydroxymethyl substituent. The starting macrocyclic diazadithiacrown ethers were obtained by treatment of a bis( $\alpha$-chloroamide) with the appropriate dimercaptan using $\mathrm{K}_{2} \mathrm{CO}_{3}$ as the base followed by reduction of the resulting macrocyclic dithiadiamide by $\mathrm{BH}_{3}-\mathrm{THF}$ or by $\mathrm{NaBH}_{4}$ in the presence of $\mathrm{BF}_{3}$-ether as a catalyst. HQ-containing ligands 23-32 were synthesized by a Mannich reaction of the secondary macrocyclic diamines with the substituted-8hydroxyquinoline. HQ-containing ligands 33-36 were prepared by reductive amination of the secondary macrocyclic diamines with 8-hydroxyquinoline-2-carbaldehyde. The HQ-containing diazadithiacrown ethers which also contain a hydroxymethyl group on the macroring 23-29, 33, and $\mathbf{3 5}$ are more soluble in polar solvents than those without the hydroxymethyl group.
\end{abstract}

Keywords: Diazadithiacrown ethers, 8-hydroxyquinoline, Mannich reaction, hydroxymethyl substituents

\section{Introduction}

In general, the complexing ability and selectivity of lariat ethers for metal ions can be varied by changing certain parameters such as the acidity of the phenolic $\mathrm{OH}$ group; the size of the crown ether ring; type, number, and position of the complexing crown ether heteroatoms; the stereochemistry imposed by the arms which connect the phenolic group to the macro ring; and 
the $\mathrm{pH}$ of media. ${ }^{1}$ For example, diaza-18-crown-6 containing two 5-chloro-8-hydroxyquinoline (CHQ) groups attached through the CHQ 7-position (1, Figure 1) exhibits a stronger affinity for $\mathrm{Mg}^{2+}$ than for $\mathrm{Ba}^{2+}\left(\log K\right.$ value in $\mathrm{MeOH}$ for $\mathrm{Mg}^{2+}$ is 6.82 , for $\left.\mathrm{Ba}^{2+} 3.60\right)$ and its isomer, diaza18-crown-6 bearing two CHQ groups attached through the CHQ 2-position 4, has a stronger affinity for $\mathrm{Ba}^{2+}$ than for $\mathrm{Mg}^{2+}$ (log $K$ value in $\mathrm{MeOH}$ for $\mathrm{Ba}^{2+}$ is 12.2). ${ }^{2}$ Ligands 3 and 5, the HQ analogs of 1 and $\mathbf{4}$, respectively ( 1 and $\mathbf{4}$ with the chlorine atoms removed), do not exhibit the same complexing properties as do $\mathbf{1}$ and $4{ }^{3}$ Increasing the number of macroring nitrogen atoms and changing the size of macroring could change the affinity of the ligand toward the heavy metal ions. For example, for ligand 5, the $\log K$ value in $\mathrm{MeOH}$ for $\mathrm{Cu}^{2+}$ is 4.39 while its tetraaza-15-crown-5 analog 6 has a $\log K$ value for $\mathrm{Cu}^{2+}$ of 15.5 .

Ligand 2, which has a 5-nitro substituent on each 8-hydroxyquinoline, has a high affinity and selectivity for $\mathrm{Hg}^{2+}$ and has proven to be a chemosensor for $\mathrm{Hg}^{2+}$. Diaza-18-crown-6 with two 4-methyl(or nitro)-6-aminophenol groups attached through the phenol 2-positions, 7 and $\mathbf{8}$ form dinuclear complexes with one $\mathrm{Cu}^{2+}$ complexed to the two 6-aminophenols and one $\mathrm{Na}^{+}$in the macroring cavity. ${ }^{6}$
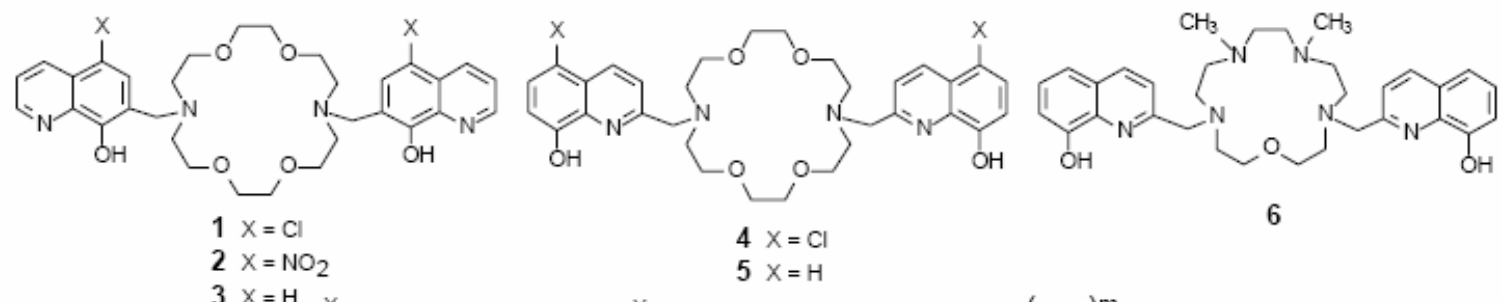

$3 \mathrm{X}=\mathrm{H}$

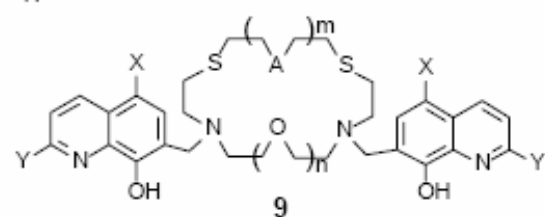

$7 \mathrm{X}=\mathrm{CH}_{3}$

$8 \times=\mathrm{NO}_{2}$

Figure 1. Compounds mentioned in the introduction.

Diazadithia (ortrithia) crown ethers 9 containing two HQ side arms have also been synthesized. ${ }^{7,8}$ These new azathia ligands have poor solubilities in $\mathrm{MeOH}$ and, therefore, their complexing properties with metal ions cannot be conveniently studied. A few of ligands $\mathbf{9}$ have a hydroxymethyl substituent attached to the macro ring and are therby more soluble in methanol. Herein, we report the synthesis of a series of new diazadithiacrown ethers bearing 5-substituent (or 2-methyl)-HQ side-arms. Some of these new ligands contain a hydroxymethyl group on the macroring. A report on the affinities of some of these new ligands for metal ions and their possible use as sensors for metal ions will be reported in due course. 


\section{Results and Discussion}

The CHQ and HQ side arms are best attached to the diazadithiacrown ethers through macroring $\mathrm{NH}$ groups. Macrocyclic ligands containing two secondary amine functions have conveniently been prepared by treating diamines and dimercaptans with various bis( $\alpha$-chloroamide)s. ${ }^{4,7,9-11}$ The $\mathrm{NH}$ functions of the secondary bis( $\alpha$-chloroamide)s are unreactive toward alkylating agents including thiols. ${ }^{7,12}$ In the present case, bis( $\alpha$-chloroamide)s 10-12 were treated with the appropriate dimercaptans using $\mathrm{K} 2 \mathrm{CO} 3$ as the base to form macrocyclic diamides 13-15 in yields of $46 \%-61 \%$ as shown in Scheme 1. The macrocyclic diamides were in turn reduced to the desired diazadithiacrown ethers 16, 17, and 22 by either $\mathrm{B}_{2} \mathrm{H}_{6}$-THF or the $\mathrm{NaBH}_{4}-\mathrm{BF}_{3}-\mathrm{THF}$ complex (Scheme 1). Ligands $\mathbf{1 8 2 1}$ shown in Scheme 1 were prepared as reported. ${ }^{7}$ Satisfactory elemental analyses were obtained for the new macrocyclic diamides or for new HQ and CHQ armed ligands 23-36 prepared from them.

Mannich aminomethylation has been used to attach HQ and CHQ groups to the azacrown ethers through HQ and CHQ 7-positions. ${ }^{3,713-15}$ In the present case, the appropriate diazadithiacrown ether 16-22 and the appropriate $\mathrm{HQ}$ derivative were treated with paraformaldehyde in refluxing benzene in the one-step aminomethylation reaction ${ }^{3,7,16,17}$ to give the bis(2-or 5-substituted-8-quinolin-7-ylmethyl)-substituted ligands 23-32 (Scheme 2).

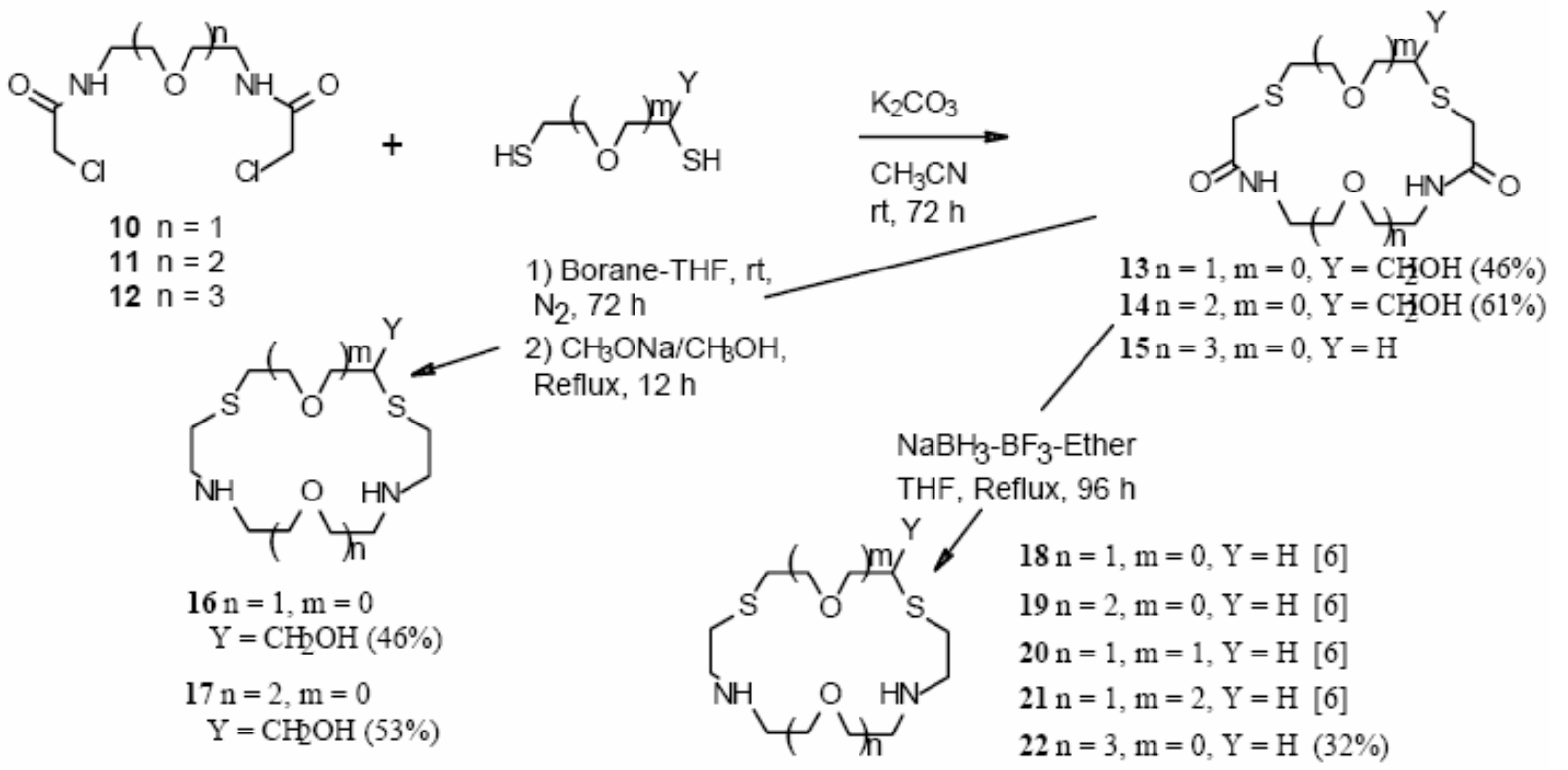

\section{Scheme 1}

The products of the Mannich reaction of the diazadithiacrown ethers with HQ (23 and 27) and 8hydroxyquinaldine (26) were mixtures. Each of these two starting materials has no substituent on the quinoline 5-position. Thus, both the 5 and 7 positions could be aminomethylated under these reaction conditions. Although we did not look for the side products 
in these reactions, we recently showed by a careful ${ }^{1} \mathrm{H}$ NMR analysis that when diazatrithiacrown ether $\mathbf{1 8}$ was treated with 8hydroxyquinaldine, the product mixture proved to be about $90 \%$ of the desired product where both quinoline substituents were attached through the 8 -hydroxyquinaldine 7 -position, about $9 \%$ of the product with one 8 -hydroxyquinaldine attached through its 7-position and the other through its 5position and the remaining product had both 8hydroxyquinaldine groups attached through their 5positions. Thus, we suspect that products 23, 26 and 27 are mixtures where the HQ groups are attached through their 7-and 5-positions.

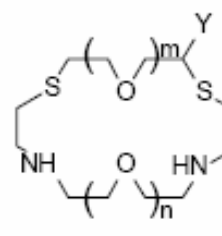

16-22
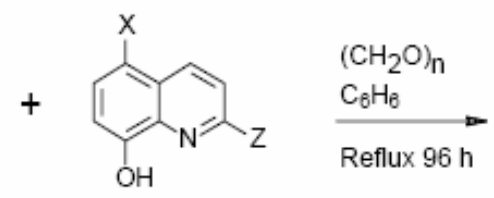

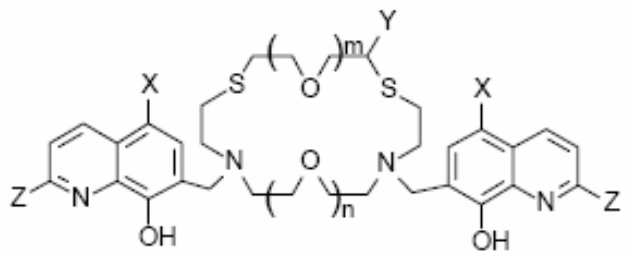

$$
\begin{aligned}
& 23 \mathrm{n}=1, \mathrm{~m}=0, \mathrm{Y}=\mathrm{CH}_{2} \mathrm{OH}, \mathrm{X}, \mathrm{Z}=\mathrm{H}(64 \%) \\
& \mathbf{2 4} \mathrm{n}=1, \mathrm{~m}=0, \mathrm{Y}=\mathrm{CH}_{2} \mathrm{OH}, \mathrm{X}=\mathrm{CH}_{3}, \mathrm{Z}=\mathrm{H}(34 \%) \\
& \mathbf{2 5} \mathrm{n}=1, \mathrm{~m}=0, \mathrm{Y}=\mathrm{CH}_{2} \mathrm{OH}, \mathrm{X}=\mathrm{Cl}, \mathrm{Z}=\mathrm{H}(57 \%) \\
& \mathbf{2 6} \mathrm{n}=1, \mathrm{~m}=0, \mathrm{Y}=\mathrm{CH}_{2} \mathrm{OH}, \mathrm{X}=\mathrm{H}, \mathrm{Z}=\mathrm{CH}_{3}(64 \%) \\
& \mathbf{2 7} \mathrm{n}=2, \mathrm{~m}=0, \mathrm{Y}=\mathrm{CH}_{2} \mathrm{OH}, \mathrm{X}, \mathrm{Z}=\mathrm{H}(64 \%) \\
& \mathbf{2 8} \mathrm{n}=2, \mathrm{~m}=0, \mathrm{Y}=\mathrm{CH}_{2} \mathrm{OH}, \mathrm{X}=\mathrm{CH} 3, \mathrm{Z}=\mathrm{H}(34 \%) \\
& \mathbf{2 9} \mathrm{n}=2, \mathrm{~m}=0, \mathrm{Y}=\mathrm{CH}_{2} \mathrm{OH}, \mathrm{X}=\mathrm{Cl}, \mathrm{Z}=\mathrm{H}(57 \%) \\
& \mathbf{3 0} \mathrm{n}=1, \mathrm{~m}=2, \mathrm{Y}=\mathrm{H}, \mathrm{X}=\mathrm{CH}_{3}, \mathrm{Z}=\mathrm{H}(56 \%) \\
& 31 \mathrm{n}=1, \mathrm{~m}=2, \mathrm{Y}=\mathrm{H}, \mathrm{X}=\mathrm{Cl}, \mathrm{Z}=\mathrm{H}(60 \%) \\
& 32 \mathrm{n}=3, \mathrm{~m}=0, \mathrm{Y}=\mathrm{H}, \mathrm{X}=\mathrm{Cl}, \mathrm{Z}=\mathrm{H}(57 \%)
\end{aligned}
$$

\section{Scheme 2}

HQ has been attached to diaza-18-crown- $6^{3}$ and a series of tetraaza-15(and 16)-crown-5 ligands ${ }^{4}$ through the HQ 2-position by a reductive amination process using $\mathrm{NaBH}(\mathrm{OAc})_{3} .{ }^{18}$ In the present case, 8-hydroxyquinoline-2-carbaldehyde and the appropriate ligand (16-18 or 20) were treated with $\mathrm{NaBH}(\mathrm{OAc})_{3}$ to form the bis(8-hydroxyquinolin-2-ylmethyl)-substituted ligands 33-36 in yields of $46 \%-66 \%$ (Scheme 3 ). It is important to note that the hydroxy group of HQ did not have to be protected for this reaction as previously reported. ${ }^{4}$ 

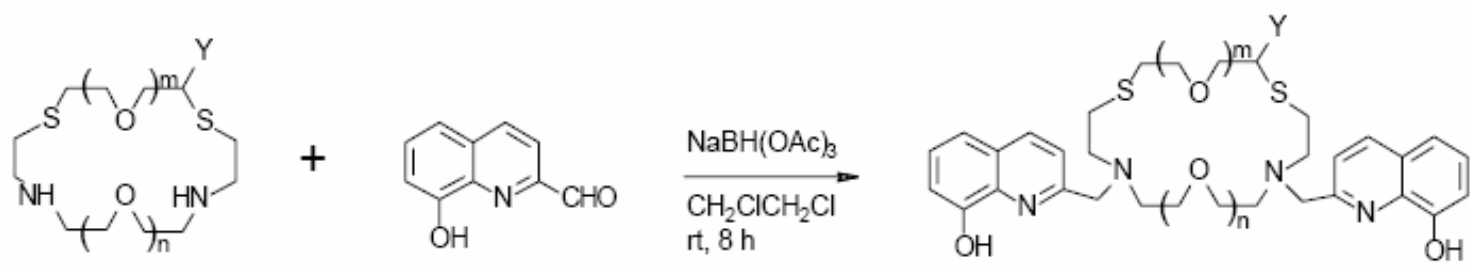

$$
\begin{aligned}
& 33 \mathrm{n}=1, \mathrm{~m}=0, \mathrm{Y}=\mathrm{CH}_{3} \mathrm{OH}(66 \%) \\
& 34 \mathrm{n}=1, \mathrm{~m}=0, \mathrm{Y}=\mathrm{H}(53 \%) \\
& 35 \mathrm{n}=2, \mathrm{~m}=0, \mathrm{Y}=\mathrm{CH}_{3} \mathrm{OH}(64 \%) \\
& 36 \mathrm{n}=1, \mathrm{~m}=1, \mathrm{Y}=\mathrm{H}(46 \%)
\end{aligned}
$$

\section{Scheme 3}

\section{Experimental Section}

General Procedures. The ${ }^{1} \mathrm{H}$ and ${ }^{13} \mathrm{C}$ NMR spectra were recorded on 200 or $300 \mathrm{MHz}$ in deuterochloroform. Solvents and starting materials were purchased from commercial sources where available. Bis ( $\alpha$-chloroamides) $\mathbf{1 0 - 1 2}{ }^{4}$ and diazadithiacrown ethers $\mathbf{1 8 - 2 1}{ }^{7}$ were prepared as reported.

General procedure A to prepare macrocyclic diamides 13-15 (Scheme 1) (,7 $^{4}$

A mixture of bis( $\alpha$-chloroamide), an equivalent of the dithiol, a 4 fold excess of anhydrous $\mathrm{K}_{2} \mathrm{CO}_{3}$ and $\mathrm{CH}_{3} \mathrm{CN}$ [350 mL/ $0.1 \mathrm{~mol}$ of the bis( $\alpha$-chloroamide)] was stirred at room temperature for $72 \mathrm{~h}$. The mixture was filtered and the solvent was evaporated under reduced pressure. The residue was separated by chromatography (silica gel, $\mathrm{C}_{2} \mathrm{H}_{4} \mathrm{Cl}_{2}: \mathrm{MeOH}: \mathrm{NH}_{4} \mathrm{OH}=80: 10: 1$ ).

11-Hydroxymethyl-1,7-diaza-4-oxa-10,13-dithiacyclopentadecan-8,15-dione (13). Macrocyclic diamide $13(2.78 \mathrm{~g}, 46 \%)$ was synthesized from $2.42 \mathrm{~g}$ (1.95 mmol) of 1,2dimercapto-3-propanol and $5.01 \mathrm{~g}(1.95 \mathrm{mmol})$ of bis ( $\alpha$-chloroamide) 10 according to general procedure A; mp 132-133.5 ${ }^{\circ} \mathrm{C}$; ${ }^{1} \mathrm{H}$ NMR: $\delta$ 2.82-3.00 (m, 3H), $3.28(\mathrm{~m}, 2 \mathrm{H}), 3,36(\mathrm{~s}, 2 \mathrm{H}), 3.47-$ $3.61(\mathrm{~m}, 8 \mathrm{H}), 3.71-3.91(\mathrm{~m}, 2 \mathrm{H}) ;{ }^{13} \mathrm{C} \mathrm{NMR}: 34.55,35.55,37.31,39.59,49.32,61.69,69.93$, 70.13, 70.54, 169.19; HRMS calcd for $\mathrm{C}_{11} \mathrm{H}_{21} \mathrm{~N}_{2} \mathrm{O}_{4} \mathrm{~S}_{2}(\mathrm{M}+\mathrm{H})^{+} 309.0944$, found 309.0938.

\section{4-Hydroxymethyl-1,10-diaza-4,7-dioxa-13,16-dithiacyclooctadecan-11,18-dione (14).}

Macrocyclic diamide 14 (8.65 g, 61\%) was obtained from $4.96 \mathrm{~g}$ (40.0 mmol) of 1,2-dimercapto3-propanol and $12.04 \mathrm{~g}(40.0 \mathrm{mmol})$ of bis( $\alpha$-chloroamide) $\mathbf{1 1}$ according to general procedure A; mp 98.5-99.5 ${ }^{\circ} \mathrm{C} ;{ }^{1} \mathrm{H}$ NMR: $\delta 2.86(\mathrm{~s}, 3 \mathrm{H}), 3.31(\mathrm{~m}, 4 \mathrm{H}), 3,73(\mathrm{~m}, 12 \mathrm{H}), 3.92(\mathrm{~m}, 2 \mathrm{H}) ;{ }^{13} \mathrm{C}$ NMR: $\delta$ 34.50, 35.49, 37.24, 39.51, 49.22, 61.67, 69.80, 70.00, 70.34, 70.43, 168.77, 168.96; HRMS calcd for $\mathrm{C}_{13} \mathrm{H}_{25} \mathrm{~N}_{2} \mathrm{O}_{5} \mathrm{~S}_{2}(\mathrm{M}+\mathrm{H})^{+} 353.1207$, found 353.1216.

1,13-Diaza-4,7,10-trioxa-16,19-dithiacycloheneicosan-14,21-dione (15). Macrocyclic dithiadiamide $15(8.76 \mathrm{~g}, 60 \%)$ was synthesized from $3.77 \mathrm{~g}(40.0 \mathrm{mmol})$ of 1,2-ethanedithiol and $13.80 \mathrm{~g}$ (40.0 mmol) of bis( $\alpha$-chloroamide) $12 ; \mathrm{mp} 106-106.5{ }^{\circ} \mathrm{C} ;{ }^{1} \mathrm{H}$ NMR: $\delta 2.81(\mathrm{~s}, 4 \mathrm{H}), 3.27$ 
(s, 4H), 3.49-3.66 (m, 16H); ${ }^{13} \mathrm{C}$ NMR: $\delta 32.71,36.30,39.57,69.65,70.63,70.82,168.68$; HRMS calcd for $\mathrm{C}_{14} \mathrm{H}_{26} \mathrm{~N}_{2} \mathrm{O}_{5} \mathrm{~S}_{2} \mathrm{Na}(\mathrm{M})^{+}$389.1147, found 389.1186; Anal. Calcd. for $\mathrm{C}_{14} \mathrm{H}_{26} \mathrm{~N}_{2} \mathrm{O}_{5} \mathrm{~S}_{2}$ : C, 45.88; H, 7.15. Found C, 45.62; H, 7.03.

General procedure B to synthesize crown ethers (16) and (17) ${ }^{4,7}$ (Scheme 1)

To a solution of $10.0 \mathrm{mmol}$ of macrocyclic diamide in $30 \mathrm{~mL}$ of dry THF was added $80 \mathrm{~mL}$ of a solution of borane in THF ( 1 mol per liter of THF). The mixture was stirred for $72 \mathrm{~h}$ at room temperature and the solvent was evaporated under reduced pressure. To this residue was added a dilute solution of $\mathrm{NaOMe}$ in $\mathrm{MeOH}$, and the mixture was refluxed overnight. After the $\mathrm{MeOH}$ was evaporated, some water was added and the mixture was extracted several times by portions of $\mathrm{CHCl}_{3}$ until all the product was extracted from the water. The combined $\mathrm{CHCl}_{3}$ extracts were dried $\left(\mathrm{Na}_{2} \mathrm{SO}_{4}\right)$, filtered, and evaporated to give the crude product which was purified by chromatography on silica gel (eluent: $\mathrm{CH}_{2} \mathrm{Cl}_{2}: \mathrm{MeOH}: \mathrm{NH}_{4} \mathrm{OH}=50: 5: 1$ ).

11-Hydroxymethyl-1,7-diaza-4-oxa-10,13-dithiacyclopentadecane (16). Ligand 16 (2.33 g, $46 \%$ ) was obtained as a low melting solid by the reduction of $2.96 \mathrm{~g}(9.6 \mathrm{mmol})$ of 13 with $38 \mathrm{~mL}$ of borane-THF complex in $30 \mathrm{~mL}$ of THF according to general procedure $\mathrm{B} ;{ }^{1} \mathrm{H} \mathrm{NMR}: \delta$ 2.79-2.89 (m, 15H), 3.59-3.65 (m, 6H); HRMS calcd for $\mathrm{C}_{11} \mathrm{H}_{25} \mathrm{~N}_{2} \mathrm{O}_{2} \mathrm{~S}_{2}(\mathrm{M}+\mathrm{H})^{+}$281.1357, found 281.1352. Anal. calcd for $\mathrm{C}_{11} \mathrm{H}_{24} \mathrm{~N}_{2} \mathrm{O}_{2} \mathrm{~S}_{2}$ : C, 47.11; H, 8.63. Found $\mathrm{C}, 47.27 ; \mathrm{H}, 8.73$.

14-Hydroxymethyl-1,10-diaz-4,7-dioxa-13,16-dithiacyclootadecane (17). Macrocyclic ether $17(0.51 \mathrm{~g}, 53 \%)$ was obtained as an oil by the reduction of $1.05 \mathrm{~g}(3.0 \mathrm{mmol})$ of 14 with $10 \mathrm{~mL}$ of borane-THF in $10 \mathrm{~mL}$ of THF according to general procedure B; ${ }^{1} \mathrm{H}$ NMR: $\delta 2.83(\mathrm{~m}, 15 \mathrm{H})$, $3.64(\mathrm{~m}, 8 \mathrm{H}), 3,78(\mathrm{~m}, 2 \mathrm{H}) ;{ }^{13} \mathrm{C} \mathrm{NMR}: \delta 31.85,33.37,35.08,48.37,49.06,49.23,49.31,64.46$, 70.02, 70.15, 70.34, 70.40; HRMS Calcd. for $\mathrm{C}_{13} \mathrm{H}_{28} \mathrm{~N}_{2} \mathrm{O}_{3} \mathrm{~S} 2 \mathrm{Na}^{+} 347.2173$, found 347.2178.

1,13-Diaza-4,7,10-trioxa-16,19-dithiacycloheneicosane (22). A solution of $2.56 \mathrm{~g}$ (7.0 mmol) of 15 and $2.64 \mathrm{~g}(70 \mathrm{mmol})$ of $\mathrm{NaBH}_{4}$ in $100 \mathrm{~mL}$ of THF was stirred at $0-5{ }^{\circ} \mathrm{C}$ and $13.2 \mathrm{~g}$ (93 mmol) of $\mathrm{BF}_{3}-\mathrm{Et}_{2} \mathrm{O}$ was added over a 3-hour period. The mixture was allowed to warm to room temperature and water was slowly added until $\mathrm{H}_{2}$ stopped evolving. The mixture was neutralized with aqueous $20 \% \mathrm{NaOH}$ to $\mathrm{pH}$ of 8 or 9 . The THF was evaporated and the aqueous solution was extracted 3 times with $20 \mathrm{~mL}$ portions of $\mathrm{CH}_{2} \mathrm{Cl}_{2}$. The combined extracts were dried $\left(\mathrm{Na}_{2} \mathrm{SO}_{4}\right)$ and the solvent was evaporated. The crude product was purified by chromatography on silica gel $\left(\mathrm{CH}_{2} \mathrm{Cl}_{2}\right.$ : $\left.\mathrm{MeOH}: \mathrm{NH}_{4} \mathrm{OH}=50: 5: 1\right)$ to give $0.76 \mathrm{~g}(32 \%)$ of $22 ;{ }^{1} \mathrm{H} \mathrm{NMR}: \delta 2.76-$ $2.82(\mathrm{~m}, 16 \mathrm{H}), 3.60-3.70(\mathrm{~m}, 12 \mathrm{H}) ;{ }^{13} \mathrm{C} \mathrm{NMR}: \delta 32.19,32.47,48.99,49.03,70.37,70.41,70.79$; HRMS calcd for $\mathrm{C}_{14} \mathrm{H}_{30} \mathrm{~N}_{2} \mathrm{O}_{3} \mathrm{~S}_{2}(\mathrm{M})^{+} 338.1699$, found 338.1691; Anal. Calcd. for $\mathrm{C}_{14} \mathrm{H}_{30} \mathrm{~N}_{2} \mathrm{O}_{3} \mathrm{~S}_{2}$ $1 / 2 \mathrm{CH}_{2} \mathrm{Cl}_{2}$ : C, 45.71; $\mathrm{H}, 8.20$; found $\mathrm{C}, 45.82 ; \mathrm{H}, 8.23$.

General procedure $C$ for the synthesis of diazadithiacrown ethers with two 5 -substitutent8-hydroxyquinoline(or 8-hydroxyquinaldine) sidearms 23-32 (Scheme 2)

A solution of $45 \mathrm{~mL}$ of anhydrous benzene, $2.0 \mathrm{mmol}$ of the macrocyclic ether, $4.2 \mathrm{mmol}$ of the 5-substitutent-8-hydroxyquinoline(or 8-hydroxyquinaldine) and paraformaldehyde (0.135 g, $4.5 \mathrm{mmol}$ ) was refluxed for $15 \mathrm{~h}$. The solvent was evaporated under reduced pressure and the 
mixture was separated by silica gel chromatography $\left(\mathrm{CH}_{2} \mathrm{Cl}_{2}: \mathrm{MeOH}: \mathrm{NH}_{4} \mathrm{OH}=50: 5: 1\right)$. 1,7-Bis(8-hydroxyquinolin-7-ylmethyl)-11-hydroxymethyl-1,7-diaza-4-oxa-10,13dithiacyclopentadecane (23). Ligand 23 (1.01 g, 65\%) was isolated as a viscous liquid from $0.73 \mathrm{~g}(2.6 \mathrm{mmol})$ of 16 and $0.83 \mathrm{~g}(5.7 \mathrm{mmol})$ of 8 -hydroxyquinoline. A small amount of hexane was added to the product and the mixture was ultrasonicated for $3 \mathrm{~h}$. After most of hexane was decanted, the remaining hexane was evaporated under reduced pressure to give a low melting solid; ${ }^{1} \mathrm{H}$ NMR: $\delta$ 2.56-2.85(m, 15H), 3.67 (t, J=4.6 Hz, 4H), 3.90-4.08 (m, 6H), 7.28 (s, 4H), 7.38 (q, $J=4.0 \mathrm{~Hz}, 2 \mathrm{H}), 8.08(\mathrm{dd}, J=1.6,1.4 \mathrm{~Hz}, 2 \mathrm{H}), 8.87(\mathrm{dd}, J=1.4,1.6 \mathrm{~Hz}, 2 \mathrm{H}) ;{ }^{13} \mathrm{C}$ NMR: $\delta 28.87,29.87,34.82,49.01,53.42,53.52,54.84,55.60,56.12,56.54,64.47,69.66$, 70.01, 119.58, 120.27, 122.28, 126.24, 127.74, 127.84, 133.14, 139.76, 149.51, 151.95, 152.09; HRMS Calcd. for $\mathrm{C}_{31} \mathrm{H}_{39} \mathrm{~N}_{4} \mathrm{O}_{4} \mathrm{~S}_{2}(\mathrm{M}+\mathrm{H})^{+}$595.2415, found 595.2411; Anal. calcd for $\mathrm{C}_{31} \mathrm{H}_{38} \mathrm{~N}_{4} \mathrm{O}_{4} \mathrm{~S}_{2}$ : C, 62.60; H, 6.44. Found: C, 62.35; H, 6.27.

1,7-Bis(5-methyl-8-hydroxyquinolin-7-ylmethyl)-11-hydroxymethyl-1,7-diaza-4-oxa-10,13dithiacyclopentadecane (24). Ligand 24 (0.32 g, 34\%) was obtained from $0.42 \mathrm{~g}(1.5 \mathrm{mmol})$ of 16 and $0.51 \mathrm{~g}(3.2 \mathrm{mmol})$ of 5-methyl-8-hydroxyquinoline. The product was purified as 23 above to give a low melting solid; ${ }^{1} \mathrm{H}$ NMR: $\delta 2.56(\mathrm{~s}, 6 \mathrm{H}), 2.78-2.96(\mathrm{~m}, 15 \mathrm{H}), 3.63(\mathrm{t}, J=3.6 \mathrm{~Hz}, 6 \mathrm{H})$, $3.94(\mathrm{~m}, 4 \mathrm{H}), 7.11(\mathrm{~d}, J=4.4 \mathrm{~Hz}, 2 \mathrm{H}), 7.40$ (q, $J=4.2 \mathrm{~Hz}, 2 \mathrm{H}), 8.21$ (dd, $J=1.2,1.6 \mathrm{~Hz}, 2 \mathrm{H})$, 8.85 (dd, $J=1.2,1.2 \mathrm{~Hz}, 2 \mathrm{H}) ;{ }^{13} \mathrm{C}$ NMR: $\delta 18.06,28.90,29.73,34.83,48.96,53.31,54.46,54.82$, $55.64,56.53,56.95,64.49,69.88,70.28,118.43,121.01,123.99,127.58,128.28,128.39,132.70$, 139.54, 148.44, 150.90, 150.99; HRMS calcd for $\mathrm{C}_{33} \mathrm{H}_{43} \mathrm{~N}_{4} \mathrm{O}_{4} \mathrm{~S}_{2}(\mathrm{M}+\mathrm{H})^{+}$623.2726, found 623.2740; Anal. Calcd. for $\mathrm{C}_{33} \mathrm{H}_{42} \mathrm{~N}_{4} \mathrm{O}_{4} \mathrm{~S}_{2}$ : C, 63.64; H, 6.80. Found C, 65.03; H,6.86.

\section{1,7-Bis(5-chloro-8-hydroxyquinolin-7-ylmethyl)-11-hydroxymethyl-1,7-diaza-4-oxa-10,13-} dithiacyclopentadecane (25). Ligand $25(0.76 \mathrm{~g}, 57 \%)$ was prepared from $0.56 \mathrm{~g}(2.0 \mathrm{mmol})$ of 16 and $0.75 \mathrm{~g}$ (4.2 mmol) of 5-chloro-8-hydroxyquinoline as 23 above; mp 133.5-134.5 ${ }^{\circ} \mathrm{C} ;{ }^{1} \mathrm{H}$ NMR: $\delta 2.77-3.08(\mathrm{~m}, 15 \mathrm{H}), 3.61(\mathrm{t}, J=3.4 \mathrm{~Hz}, 6 \mathrm{H}), 3.95(\mathrm{~s}, 4 \mathrm{H}), 7.44(\mathrm{~d}, J=10.2 \mathrm{~Hz}, 2 \mathrm{H}), 7.50$ (q, $J=3.8 \mathrm{~Hz}, 2 \mathrm{H}), 8.46(\mathrm{dd}, J=1.8,1.8 \mathrm{~Hz}, 2 \mathrm{H}), 8.90$ (dd, $J=1.5,1.5 \mathrm{~Hz}, 2 \mathrm{H})$; HRMS calcd for $\mathrm{C}_{31} \mathrm{H}_{37} \mathrm{Cl}_{2} \mathrm{~N}_{4} \mathrm{O}_{4} \mathrm{~S}_{2}(\mathrm{M}+\mathrm{H})^{+}$663.1637, found 663.1615; Anal. calcd for $\mathrm{C}_{31} \mathrm{H}_{36} \mathrm{Cl}_{2} \mathrm{~N}_{4} \mathrm{O}_{4} \mathrm{~S}_{2}$ : C, 56.10; H, 5.47. Found: C, 55.92; H, 5.61

\section{1,7-Bis(2-methyl-8-hydroxyquinolin-7-ylmethyl)-11-hydroxymethyl-1,7-diaza-4-oxa-10,13-}

dithiacyclopentadecane (26). Compound $26(0.26 \mathrm{~g}, 64 \%)$ was obtained as a low melting solid from $0.28 \mathrm{~g}(1.0 \mathrm{mmol})$ of $\mathbf{1 6}$ and $0.33 \mathrm{~g}(2.1 \mathrm{mmol})$ of 8 -hydroxyquinaldine as 23 above; ${ }^{1} \mathrm{H}$ NMR: $\delta 2.78(\mathrm{~s}, 6 \mathrm{H}), 2.85-3.10(\mathrm{~m}, 15 \mathrm{H}), 3.61-3.80(\mathrm{~m}, 6 \mathrm{H}), 3.86-3.98(\mathrm{~m}, 4 \mathrm{H}), 7.23(\mathrm{~d}, J=3.7$ $\mathrm{Hz}, 4 \mathrm{H}), 7.28$ (d, $J=1.6 \mathrm{~Hz}, 2 \mathrm{H}), 8.00$ (d, $J=8.6 \mathrm{~Hz}, 2 \mathrm{H}) ;{ }^{13} \mathrm{C}$ NMR: $\delta 25.43,25.50,28.79,29.61$, 34.77, 48.81, 53.14, 53.36, 54.84, 55.82, 56.41, 56.85, 64.73, 69.92, 70.42, 117.53, 119.07, $122.53,126.83,127.24,127.35,136.23,138.71,152.22,157.99$; Anal. calcd for $\mathrm{C}_{33} \mathrm{H}_{42} \mathrm{~N}_{4} \mathrm{O}_{4} \mathrm{~S}_{2}$ : C, 63.64; H, 6.80. Found: C, 63.76; H, 6.68.

1,10-Bis(8-hydroxyquinolin-7-ylmethyl)-14-hydroxymethyl-1,10-diaza-4,7-dioxa-13,16dithiacyclooctadecane (27). Ligand $27(0.72 \mathrm{~g}, 64 \%)$ was obtained from $0.65 \mathrm{~g}(2.0 \mathrm{mmol})$ of 17 and $0.61 \mathrm{~g}$ (4.2 mmol) of 8hydroxyquinoline. It was purified as 23 above to give a low melting solid; ${ }^{1} \mathrm{H}$ NMR: $\delta$ 2.70-2.95 (s, 15H), 3.62-3.65 (m, 10H), $3.95(\mathrm{~d}, 4 \mathrm{H}), 7.27(\mathrm{~s}, 4 \mathrm{H})$, 
7.38 (q, $J=4.0 \mathrm{~Hz}, 2 \mathrm{H}), 8.06(\mathrm{dd}, J=1.6,1.4 \mathrm{~Hz}, 2 \mathrm{H}), 8.86(\mathrm{dd}, J=1.4,1.6 \mathrm{~Hz}, 2 \mathrm{H})$; HRMS for $\mathrm{C}_{33} \mathrm{H}_{43} \mathrm{~N}_{4} \mathrm{O}_{5} \mathrm{~S}_{2}(\mathrm{M}+\mathrm{H})^{+}$639.2675, found 639.2666; Anal. calcd for $\mathrm{C}_{33} \mathrm{H}_{42} \mathrm{~N}_{4} \mathrm{O}_{5} \mathrm{~S}_{2}$ : C, 62.04; $\mathrm{H}$, 6.63. Found: C, 61.88; H, 6.45 .

\section{1,10-Bis(5-methyl-8-hydroxyquinolin-7-ylmethyl)-14-hydroxymethyl-1,10-diaza-4,7-dioxa-} 13,16-dithiacyclooctadecane (28). Ligand 28 (0.98 g, 94\%) was synthesized from $0.51 \mathrm{~g}$ $(1.57 \mathrm{mmol})$ of 17 and $0.52 \mathrm{~g}(3.30 \mathrm{mmol})$ of 5-methyl-8-hydroxyquinoline as a viscous liquid. The product was purified as 23 above to give a white powder; mp 59-60 ${ }^{\circ} \mathrm{C}$; ${ }^{1} \mathrm{H}$ NMR: $\delta 2.55$ (s, $6 \mathrm{H}), 2.74-2.99(\mathrm{~m}, 15 \mathrm{H}), 3.61-3.63(\mathrm{~m}, 10 \mathrm{H}), 3.94(\mathrm{~d}, 4 \mathrm{H}), 7.10(\mathrm{~d}, J=3.3 \mathrm{~Hz}, 2 \mathrm{H}), 7.38$ (q, $J=4.2 \mathrm{~Hz}, 2 \mathrm{H}), 8.20(\mathrm{~d}, J=5.4 \mathrm{~Hz}, 2 \mathrm{H}), 8.85(\mathrm{t}, J=2.4 \mathrm{~Hz}, 2 \mathrm{H})$; HRMS calcd for $\mathrm{C}_{35} \mathrm{H}_{47} \mathrm{~N}_{4} \mathrm{O}_{5} \mathrm{~S}_{2}(\mathrm{M}+\mathrm{H})^{+}$667.2991, found 667.2997; Anal. Calcd. for $\mathrm{C}_{35} \mathrm{H}_{46} \mathrm{~N}_{4} \mathrm{O}_{5} \mathrm{~S}_{2}$ : C, 63.03; $\mathrm{H}$, 6.95. Found: C, 62.89; H, 7.13.

\section{1,10-Bis(5-chloro-8-hydroxyquinolin-7-ylmethyl)-14-hydroxymethyl-1,10-diaza-4,7-dioxa-}

13,16-dithiacyclooctadecane (29). Ligand $29(0.31 \mathrm{~g}, 28 \%)$ was prepared from $0.51 \mathrm{~g}$ $(1.57 \mathrm{mmol})$ of 17 and $0.59 \mathrm{~g}(3.30 \mathrm{mmol})$ of 5-chloro-8-hydroxyquinoline as a viscous liquid. It was purified as 23 above; ${ }^{1} \mathrm{H}$ NMR: $\delta 2.76-3.02(\mathrm{~m}, 15 \mathrm{H}), 3.67-3.73(\mathrm{~m}, 10 \mathrm{H}), 3.99(\mathrm{~d}, 4 \mathrm{H}), 7.43$ (d, J=2.6 Hz, 2H), 7.49 (q, J=4.0 Hz, 2H), 8.47 (dd, J=2.0, 1.6 Hz, 2H), 8.89 (dd, J=1.4, $1.8 \mathrm{~Hz}$, $2 \mathrm{H}$ ); HRMS calcd for $\mathrm{C}_{33} \mathrm{H}_{41} \mathrm{Cl}_{2} \mathrm{~N}_{4} \mathrm{O}_{5} \mathrm{~S}_{2}(\mathrm{M}+\mathrm{H})^{+} 707.1899$, found 707.1880; Anal. calcd for $\mathrm{C}_{33} \mathrm{H}_{40} \mathrm{Cl}_{2} \mathrm{~N}_{4} \mathrm{O}_{5} \mathrm{~S}_{2}$ : C, 56.00; H, 5.70. Found: C, 56.12; H, 5.58.

\section{1,7-Bis(5-methyl-8-hydroxyquinolin-7-ylmethyl)-1,7-diaza-4,13,16-trioxa-}

10,19-dithiacycloheneicosane (30). According to general procedure $C$, ligand 30 (56\%) was synthesized from 21 and 5-methyl-8-hydroxyquinoline as a viscous liquid. It was treated as 23 above; ${ }^{1} \mathrm{H}$ NMR: $\delta 2.55(\mathrm{~s}, 6 \mathrm{H}), 2.72(\mathrm{t}, J=6.2 \mathrm{~Hz}, 4 \mathrm{H}), 2.90(\mathrm{~m}, 12 \mathrm{H}), 3.66(\mathrm{~m}, 12 \mathrm{H}), 3.94(\mathrm{~s}$, 4H), 7.03 (s, 2H), 7.38 (q, J=4.0 Hz, 2H), 8.20 (dd, J=1.4, 1.4 Hz, 2H), 8.86 (dd, J=1.4, 1.4 Hz, 2H); ${ }^{13} \mathrm{C}$ NMR: $\delta 17.97,29.47,31.48,53.06,54.55,56.43,69.50,70.67,71.79,118.36,120.83$, 123.81, 127.43, 128.22, 132.49, 139.52, 148.31, 150.98; Anal. calcd for $\mathrm{C}_{36} \mathrm{H}_{48} \mathrm{~N}_{4} \mathrm{O}_{5} \mathrm{~S}_{2}$ : C, 63.50, H, 7.11. Found: C, 63.32, H, 7.28.

\section{1,7-Bis(5-chloro-8-hydroxyquinolin-7-ylmethyl)-1,7-diaza-4,13,16-trioxa-10,} 19-dithiacycloheneicosane (31). According to general procedure $\mathrm{C}$, ligand 31 (0.65 g, 60\%) was synthesized from $0.51 \mathrm{~g}(1.5 \mathrm{mmol})$ of 21 and 5-chloro-8-hydroxyquinoline as a viscous liquid; ${ }^{1} \mathrm{H}$ NMR: $\delta 2.73(\mathrm{t}, J=6.2 \mathrm{~Hz}, 4 \mathrm{H}), 2.92(\mathrm{~m}, 12 \mathrm{H}), 3.67(\mathrm{~m}, 12 \mathrm{H}), 3.98(\mathrm{~s}, 4 \mathrm{H}), 7.41$ (s, 2H), 7.48 (m, 2H), 8.45 (dd, $J=1.6,1.4 \mathrm{~Hz}, 2 \mathrm{H}), 8.90$ (dd, $J=1.4,1.4 \mathrm{~Hz}, 2 \mathrm{H}) ;{ }^{13} \mathrm{C}$ NMR: $\delta 29.44$, $31.40,52.98,54.41,55.99,69.27,70.58,71.75,119.37,119.88,121.93,125.91,127.51,132.72$, 139.57, 149.18, 151.92; Anal. calcd for $\mathrm{C}_{34} \mathrm{H}_{42} \mathrm{Cl}_{2} \mathrm{~N}_{4} \mathrm{O}_{5} \mathrm{~S}_{2}: \mathrm{C}, 56.58, \mathrm{H}, 5.87$. Found: C, 56.76, $\mathrm{H}$, 5.69 .

\section{1,13-Bis(5-chloro-8-hydroxyquinolin-7-ylmethyl)-1,13-diaz-4,7,10-trioxa-}

16,19-dithiacycloheneicosane (32). Ligand 32 (57\%) was isolated as a viscous liquid from marocyclic diamine 22 and 5-chloro-8-hydroxyquinoline; ${ }^{1} \mathrm{H}$ NMR: $\delta$ 2.77-2.99 $(\mathrm{m}, 16 \mathrm{H}), 3.67-$ $3.71(\mathrm{~m}, 12 \mathrm{H}), 3.97(\mathrm{~s}, 4 \mathrm{H}), 7.41(\mathrm{~s}, 2 \mathrm{H}), 7.49$ (q, J=4.4 Hz, 2H), 8.46 (dd, J=1.4, $1.4 \mathrm{~Hz}, 2 \mathrm{H})$, $8.90(\mathrm{dd}, J=1.4,1.8 \mathrm{~Hz}, 2 \mathrm{H}) ;{ }^{13} \mathrm{C}$ NMR: $\delta 29.31,31.76,32.33,53.17,54.42,56.53,69.61,70.89$, $119.48,120.19$, 122.18, 126.17, 127.75, 133.00, 139.81, 149.41, 152.06; HRMS Calcd. for 
$\mathrm{C}_{34} \mathrm{H}_{43} \mathrm{Cl}_{2} \mathrm{~N}_{4} \mathrm{O}_{5} \mathrm{~S}_{2}(\mathrm{M}+\mathrm{H})^{+}$721.2056, found 721.2042; Anal. calcd for $\mathrm{C}_{34} \mathrm{H}_{42} \mathrm{Cl}_{2} \mathrm{~N}_{4} \mathrm{O}_{5} \mathrm{~S}_{2}$ : C, 56.58, $\mathrm{H}, 5.87$, found. C, 56.38, H, 6.00.

General procedure $D$ for the reductive amination of azathiacrown ethers with 8Hydroxyquinoline-2-carbaldehyde ${ }^{3,4}$ to form ligands 33-36 (Scheme 3)

The crown ether $(3.0 \mathrm{mmol})$ and $1.08 \mathrm{~g}(6.3 \mathrm{mmol})$ of 8-hydroxyquinoline-2-carbaldehyde were added to $60 \mathrm{~mL}$ of 1,2-dichloroethane and the mixture was stirred at room temperature for $30 \mathrm{~min} . \mathrm{NaBH}(\mathrm{OAc})_{3}(1.92 \mathrm{~g}, 9.0 \mathrm{mmol})$ was added and the mixture was stirred overnight at room temperature. Saturated $\mathrm{NaHCO}_{3}(50 \mathrm{~mL})$ was then added to quench the reaction. The aqueous and organic layers were separated and the aqueous layer was extracted three times with $20 \mathrm{~mL}$ portions of $\mathrm{CH}_{2} \mathrm{Cl}_{2}$. The combined organic extracts were dried over $\mathrm{Na}_{2} \mathrm{SO}_{4}$. The solvent was evaporated under reduced pressure and the product was separated by silica gel chromatography $\left(\mathrm{CH}_{2} \mathrm{Cl}_{2}: \mathrm{MeOH}: \mathrm{NH}_{4} \mathrm{OH}=80: 5: 1\right)$.

\section{1,7-Bis(8-hydroxyquinolin-2-ylmethyl)-11-hydroxymethyl-1,7-diaza-4-oxa-10,13-}

dithiacyclooctadecane (33). Ligand 33 (1.18 g, 66\%) was prepared from 16 according to general procedure $\mathrm{D}$. The crude product was purified by chromatography; mp $140.5-142.0^{\circ} \mathrm{C}$; ${ }^{1} \mathrm{H}$ NMR: $\delta$ 2.80-3.08(m, 15H), $3.54(\mathrm{~m}, 6 \mathrm{H}), 3.96(\mathrm{~s}, 4 \mathrm{H}), 7.16(\mathrm{~d}, J=5.0 \mathrm{~Hz}, 2 \mathrm{H}), 7.31(\mathrm{~s}, 2 \mathrm{H})$, $7.41(\mathrm{t}, J=7.6 \mathrm{~Hz}, 2 \mathrm{H}), 7.61(\mathrm{t}, J=8.4 \mathrm{~Hz}, 2 \mathrm{H}), 8.12$ (d, $J=8.4 \mathrm{~Hz}, 2 \mathrm{H}) ;{ }^{13} \mathrm{C}$ NMR: $\delta 29.29,30.47$, 35.42 , 48.59, 54.26, 55.62, 55.96, 61.45, 64.25, 70.36, 70.57, 110.50, 117.72, 121.96, 127.45, 127.65, 136.58, 152.22, 152.46, 158.03; HRMS calcd for $\mathrm{C}_{31} \mathrm{H}_{39} \mathrm{~N}_{4} \mathrm{O}_{4} \mathrm{~S}_{2}(\mathrm{M}+\mathrm{H})^{+} 595.2415$, found 595.2419; Anal. Calcd. for $\mathrm{C}_{31} \mathrm{H}_{38} \mathrm{~N}_{4} \mathrm{O}_{4} \mathrm{~S}_{2}$ : C, 62.60; H, 6.44. Found: C, 62.67; H, 6.30.

\section{1,10-Bis(8-hydroxyquinolin-2-ylmethyl)-1,10-diaza-4,7-dioxa-13,16-dithia-cyclooctadecane}

(34). Ligand 34 (53\%) was synthesized from 18 as a viscous liquid according to procedure D. It was treated as 23 above to give a low melting solid; ${ }^{1} \mathrm{H}$ NMR: $\delta 2.84(\mathrm{~m}, 16 \mathrm{H}), 3.60(\mathrm{~s}, 8 \mathrm{H}), 3.97$ (s, 4H), $7.12(\mathrm{~d}, J=6 \mathrm{~Hz}, 2 \mathrm{H}), 7.39(\mathrm{~m}, 2 \mathrm{H}), 7.65(\mathrm{~m}, 4 \mathrm{H}), 8.09$ (m, 2H); HRMS Calcd. for $\mathrm{C}_{32} \mathrm{H}_{41} \mathrm{~N}_{4} \mathrm{O}_{4} \mathrm{~S}_{2}(\mathrm{M}+\mathrm{H})^{+}$609.2572, found 609.2560; Anal. calcd for $\mathrm{C}_{32} \mathrm{H}_{40} \mathrm{~N}_{4} \mathrm{O}_{4} \mathrm{~S}_{2}$ : C, 63.13; $\mathrm{H}$, 6.62. Found: C, 62.92; H, 6.86 .

\section{1,10-Bis(8-hydroxyquinolin-2-ylmethyl)-14-Hydroxymethyl-1,10-diaza-4,7-dioxa-13,16-}

dithiacyclooctadecane (35). Compound 35 (64\%) was obtained as a viscous liquid from 17 according to general procedure $\mathrm{D} . \mathrm{CH}_{2} \mathrm{Cl}_{2}(1 \mathrm{~mL})$ was added to dissolve the crude product, then $15 \mathrm{~mL}$ of hexane was added and the product was purified as 23 above to obtain a low melting solid; ${ }^{1} \mathrm{H}$ NMR: $\delta$ 2.84-3.00 (m, 15H), 3.61-3.66 (m, 8H), $3.96(\mathrm{~m}, 6 \mathrm{H}), 7.17(\mathrm{~d}, J=6.8 \mathrm{~Hz}, 2 \mathrm{H})$, 7.30 (t, $J=1.8 \mathrm{~Hz}, 2 \mathrm{H}), 7.41(\mathrm{~d}, J=7.8 \mathrm{~Hz}, 2 \mathrm{H}), 7.53(\mathrm{~m}, 2 \mathrm{H}), 8.08(\mathrm{dd}, J=2.6,2.2 \mathrm{~Hz}, 2 \mathrm{H}) ;{ }^{13} \mathrm{C}$ NMR: $\delta$ 29.22, 30.50, 35.35, 48.91, 54.21, 54.32, 55.40, 55.78, 61.71, 64.31, 65.02, 69.91, $70.05,70.71,110.42,110.80,117.67,119.40,121.85,121.98,127.41,127.50,127.74,127.80$, 136.61, 136.66, 137.67, 137.96, 152.39, 152.81, 157.37, 158.08; HRMS for $\mathrm{C}_{33} \mathrm{H}_{43} \mathrm{~N}_{4} \mathrm{O}_{5} \mathrm{~S}_{2}(\mathrm{M}+\mathrm{H})^{+}$639.2678, found 639.2682; Anal. Calcd. for $\mathrm{C}_{33} \mathrm{H}_{42} \mathrm{~N}_{4} \mathrm{O}_{5} \mathrm{~S}_{2}$ : C, 62.04; $\mathrm{H}$, 6.63. Found: C, 61.88; H, 6.45. 


\section{1,7-Bis(8-hydroxyquinolin-2-ylmethyl)1,7-diaza-4,13-dioxa-10,16-dithiacyclootadecane}

(36). Ligand 36 (46\%) was synthesized from 20 according to procedure D; ${ }^{1} \mathrm{H}$ NMR: $2.73-2.92$ (m, 16H), 3.47-3.55 (m, 4H), 3.66-3.72 (m, 4H), $3.96(\mathrm{~s}, 4 \mathrm{H}), 7.12(\mathrm{dd}, J=1.2,1.4 \mathrm{~Hz}, 2 \mathrm{H}), 7.27$ $(\mathrm{m}, 2 \mathrm{H}), 7.37$ (dd, $J=2.8,2.6 \mathrm{~Hz}, 2 \mathrm{H}), 7.64(\mathrm{dd}, J=3.0,2.6 \mathrm{~Hz}, 2 \mathrm{H}), 8.06$ (dd, $J=2.6,2.6 \mathrm{~Hz}, 2 \mathrm{H})$; ${ }^{13} \mathrm{C}$ NMR: $\delta 30.37,31.67,53.81,55.00,61.51,69.91,71.87,110.14,117.68,121.97,127.28$, 127.63, 136.51, 137.49, 152.19, 158.28; Anal. calcd for $\mathrm{C}_{32} \mathrm{H}_{40} \mathrm{~N}_{4} \mathrm{O}_{4} \mathrm{~S}_{2}$ : C, 63.13; $\mathrm{H}, 6.62$. Found: C, 62.95; H, 6.61.

\section{Acknowledgements}

The authors thank the U.S. Office of Naval Research and the Chinese Scientific and Technical Commission of Guangzhou for Financial support.

\section{References}

1. (a) van Verggel, F. C. J. M.; Verboom, W.; Reinhoudt, D. N. Chem Rev. 1994, 94, 279. (b) Graf, E.; Hosseini, M. W.; De Cian, A.; Fischer, J. Bull. Soc. Chim. Fr. 1996, 133, 743. (c) Gokel, G. W.; Schall, O. F. In Comprehensive Supramolecular Chemistry, Gokel, G. W., Ed.; Pergamon: New York, 1996; Vol. 1, p 97. (d) Bartsch, R. A.; Bitalac, L. P.; Cowey, C. L.; Elshani, S.; Goo, M.-J.; Huber, V. J.; Ivy, S. N.; Jang, Y.; Johnson, R. J.; Kim, J. S.; Luboch, E.; McDonough, J. A.; Pugia, M. J.; Son, B.; Zhao, Q. J Heterocyclic Chem. 2000, 37, 1337. (e) Habata, Y.; Saeki, T.; Akabori, S.; Zhang, X. X.; Bradshaw, J. S. J. Heterocylic Chem. 2001, 38, 253.

2. Zhang, X. X.; Bordunov, A. V.; Bradshaw, J. S.; Dalley, N. K.; Kou, X.; Izatt, R. M. J. Am. Chem. Soc. 1995, 117, 11507.

3. Su, N.; Bradshaw, J. S.; Zhang, X. X.; Song, H.-C.; Xue, G.-P.; Savage, P. B.; Krakowiak, K. E.; Izatt, R. M. J. Org. Chem. 1999, 64, 8855.

4. Yang, Z.; Bradshaw, J. S.; Zhang, X. X.; Savage, P. B.; Krakowiak, K. E.; Dalley, N. K.; Su, N.; Bronson, R. T.; Izatt, R. M. J. Org. Chem. 1999, 64, 3162.

5. Prodi, L.; Bargossi, C.; Montalti, M.; Zaccheroni, N.; Su, N.; Bradshaw J. S.; Izatt, R. M.; Savage, P.B. J. Am. Chem. Soc. 2000, 122, 6769.

6. Su, N.; Bradshaw, J. S.; Zhang, X. X.; Savage, P. B.; Krakowiak, K. E.; Izatt, R. M. J. Org. Chem. 1999, 64, 3825.

7. Bradshaw J. S.; Song H-C; Xue G-P, Bronson T. R.; Chiara J. A.; Krakowiak K. E.; Savage P. B.; Izatt R. M. Supramol. Chem. in press.

8. Xue G-P; Bradshaw J. S.; Song H-C; Bronson T. R.; Savage P. B.; Krakowiak K. E.; Izatt R. M.; Prodi L.; Montalti M.; Zaccheroni N. Tetrahedron 2001, 57, 87.

9. Bradshaw, J. S.; Krakowiak, K. E.; Izatt, R. M. J. Heterocyclic Chem. 1989, 26, 1431. 
10. Krakowiak, K. E.; Bradshaw, J. S.; Izatt, R. M. J. Org. Chem. 1990, 55, 3364.

11. Krakowiak, K. E.; Bradshaw, J. S.; Izatt, R. M. Synlett 1993, 611.

12. Bradshaw, J. S.; Krakowiak, K. E.; An, H.-Y.; Izatt, R. M. J. Heterocyclic Chem. 1990, 27, 2113.

13. Lukyanenko, N. K.; Pastushok, A.N.; Bordunov, A.V.; Vetrogon, V.I.; Vetrogon, N.I.; Bradshaw, J.S. J. Chem. Soc., Perkin Trans. 1 1994, 1489.

14. Bordunov, A. V.; Bradshaw, J. S.; Zhang, X. X.; Dalley, N. K.; Kou, X.; Izatt, R. M. Inorg. Chem. 1996, 35, 7229.

15. Bordunov, A. V.; Bradshaw, J. S.; Pastushok, V. N; Izatt, R. M. Synlett 1996, 933

16. Chi, K.-W.; Wei, H.-C.; Kottke, T.; Lagow, R. J. J. Org. Chem. 1996, 61, 5684.

17. Su, N.; Bradshaw, J. S.; Savage, P. B.; Krakowiak, K. E.; Izatt, R. M.; DeWall, S. L.; Gokel, G. W. Tetrahedron 1999, 55, 9737.

18. Abdel-Magid, A. F.; Carson, K. G.; Harris, B. D.; Maryanoff, C. A.; Shah, R. D. J. Org. Chem. 1996, 61, 3849. 